\title{
Tripled Coincidence and Common Fixed Point Results for Two Pairs of Hybrid Mappings
}

\author{
Marwan Amin Kutbi, ${ }^{1}$ Jamshaid Ahmad, ${ }^{2}$ Mujahid Abbas, ${ }^{3}$ and Muhammad Arshad ${ }^{4}$ \\ ${ }^{1}$ Department of Mathematics, King Abdul Aziz University, Jeddah, Saudi Arabia \\ ${ }^{2}$ Department of Mathematics, COMSATS Institute of Information Technology, Chack Shahzad, Islamabad 44000, Pakistan \\ ${ }^{3}$ Department of Mathematics \& Applied Mathematics, University of Pretoria, Pretoria 002, South Africa \\ ${ }^{4}$ Department of Mathematics, International Islamic University, H-10, Islamabad 44000, Pakistan
}

Correspondence should be addressed to Muhammad Arshad; marshad_zia@yahoo.com

Received 26 August 2013; Accepted 24 December 2013; Published 30 January 2014

Academic Editor: Patricia J. Y. Wong

Copyright (C) 2014 Marwan Amin Kutbi et al. This is an open access article distributed under the Creative Commons Attribution License, which permits unrestricted use, distribution, and reproduction in any medium, provided the original work is properly cited.

The tripled fixed point is a generalization of the well-known concept of "coupled fixed point." In this paper, tripled coincidence and common fixed point results for two hybrid pairs consisting of multivalued and single valued mappings on a metric space are proved. We give examples to illustrate our results. In the process, several comparable coincidence and fixed point results in the existing literature are improved, unified, and generalized.

\section{Introduction and Preliminaries}

The study of fixed points for multivalued contraction mappings using the Hausdorff metric was initiated by Nadler Jr. [1]. After this, fixed point theory has been developed further and applied to many disciplines to solve functional equations. Banach contraction principle has been extended in different directions. Some authors used generalized contractions for multivalued mappings and hybrid pairs of single and multi-valued mappings, while others used more general spaces. Dhage [2,3] established hybrid fixed point theorems and obtained some applications of presented results. Gnana Bhaskar and Lakshmikantham [4] introduced the notion of a coupled fixed point and proved some coupled fixed point results under certain contractive conditions in a complete metric space endowed with a partial order. They applied their results to study the existence and uniqueness of solution for a periodic boundary value problem associated with a firstorder ordinary differential equation. Later, Lakshmikantham and Ćirić [5] established the existence of coupled coincidence point results to generalize the results of Gnana Bhaskar and Lakshmikantham [4]; Karapınar [6] generalized these results on a complete cone metric space endowed with a partial order. Recently, Berinde and Borcut $[7,8]$ introduced the concept of a tripled fixed point for nonlinear contractive mappings in partially ordered complete metric spaces and obtained tripled coincidence and fixed point results for commuting maps. Hussain et al. $[9,10]$ obtained some coupled and tripled coincidence results without compatibility. Ilić et al. [11] obtained coupled coincidence and common fixed point theorems for a hybrid pair of mappings. For other related results in this direction, we refer to [12-16] and references mentioned therein. The purpose of this paper is to obtain tripled coincidence and common fixed point results for two hybrid pairs consisting of multivalued and single valued mappings.

Let us recall some definitions and well known results needed in the sequel.

Let $(X, d)$ be a metric space. For $x \in X$ and $A \subseteq X$, we denote $d(x, A)=\inf \{d(x, y): y \in A\}$. The set of all nonempty bounded and closed subsets of $X$ is denoted by $C B(X)$. Let $H$ be the Hausdorff metric induced by the metric $d$ on $X$; that is,

$$
H(A, B)=\max \left\{\sup _{x \in A} d(x, B), \sup _{y \in B} d(y, A)\right\},
$$

for every $A, B \in C B(X)$. 
Lemma 1 (see [1]). Let $A, B \in C B(X)$ and $\alpha>1$. Then, for every $a \in A$, there exists $b \in B$ such that

$$
d(a, b) \leq \alpha H(A, B) .
$$

Lemma 2 (see [1]). Let $A, B \in C B(X)$ and $0<\alpha \in \mathbb{R}$. Then, for every $a \in A$, there exists $b \in B$ such that

$$
d(a, b) \leq H(A, B)+\alpha .
$$

Lemma 3 (see [1]). Let $A, B \in C B(X)$. If $a \in A$, then $d(a, B) \leq$ $H(A, B)$.

Definition 4. Let $X$ be a nonempty set, $F: X \times X \times X \rightarrow 2^{X}$ (collection of all nonempty subsets of $X$ ) and $g: X \rightarrow X$. An element $(x, y, z) \in X \times X \times X$ is called (i) a tripled fixed point of $F$ if $x \in F(x, y, z), y \in F(y, z, x)$, and $z \in F(z, x, y)$ (ii) tripled coincidence point of a hybrid pair $(F, g)$ if $g(x) \in$ $F(x, y, z), g(y) \in F(y, z, x)$ and $g(z) \in F(z, x, y)$ (iii) tripled common fixed point of a hybrid pair $(F, g)$ if $x=g(x) \epsilon$ $F(x, y, z), y=g(y) \in F(y, z, x)$, and $z=g(z) \in F(z, x, y)$.

We denote the set of tripled coincidence point of a hybrid pair $(F, g)$ by $\Upsilon(F, g)$. Note that if $(x, y, z) \in \Upsilon(F, g)$, then $(y, z, x)$ and $(z, x, y)$ are also in $\Upsilon(F, g)$.

Definition 5. Let $F: X \times X \times X \rightarrow 2^{X}$ and $g: X \rightarrow X$. Then the hybrid pair $(F, g)$ is called $w$-compatible if $g(F(x, y, z)) \subseteq$ $F(g x, g y, g z)$ whenever $(x, y, z) \in \Upsilon(F, g)$.

Definition 6. Let $F: X \times X \times X \rightarrow 2^{X}$ and $g: X \rightarrow X$. The mapping $g$ is called $F$-idempotent at some point $(x, y, z) \in$ $X \times X \times X$ if $g^{2}(x) \in F(g x, g y, g z), g^{2}(y) \in F(g y, g z \cdot g x)$, and $g^{2}(z) \in F(g z, g x \cdot g y)$.

\section{Main Result}

Theorem 7. Let $(X, d)$ be a metric space, $S, T: X \times X \times X \rightarrow$ $C B(X)$ and let $g: X \rightarrow X$ be mappings such that

$$
\begin{aligned}
H(S & (x, y, z), T(u, v, w)) \\
\leq & a_{1} d(g x, g u)+a_{2} d(g y, g v)+a_{3} d(g z, g w) \\
& +a_{4} d(S(x, y, z), g x)+a_{5} d(T(u, v, w), g u) \\
& +a_{6} d(S(x, y, z), g u)+a_{7} d(T(u, v, z), g x),
\end{aligned}
$$

for all $x, y, z, u, v, w \in X$, where $a_{i}=a_{i}(x, y, z, u, v, w), i=$ $1,2, \ldots, 7$, are nonnegative real such that

$$
a_{1}+a_{2}+a_{3}+a_{4}+a_{5}+a_{6}+a_{7} \leq h<1 .
$$

If $S(X \times X \times X) \cup T(X \times X \times X) \subseteq g(X)$ and $g(X)$ is complete subset of $X$, then $(S, g)$ and $(T, g)$ have tripled coincidence point. Moreover $(S, g)$ and $(T, g)$ have tripled common fixed point if one of the following conditions holds:

(i) $(S, g)$ and $(T, g)$ are $w$-compatible, $\lim _{n \rightarrow \infty} g^{n} x=u$, $\lim _{n \rightarrow \infty} g^{n} y=v$ and $\lim _{n \rightarrow \infty} g^{n} z=w$ for some $(x, y, z) \in \Upsilon(S, g) \cap \Upsilon(T, g), u, v, w \in X$ and $g$ is continuous at $u, v, w$; (ii) if $g^{2} x=g x, g^{2} y=g y$, and $g^{2} z=g z$ and $g$ is $S, T$ idempotent for $(x, y, z) \in \Upsilon(S, g) \cap \Upsilon(T, g)$;

(iii) $g$ is continuous at $x, y, z$ for some $(x, y, z) \in \Upsilon(g, S) \cap$ $\Upsilon(g, T)$ and for some $u, v, w \in X$; $\lim _{n \rightarrow \infty} g^{n} u=x$; and $\lim _{n \rightarrow \infty} g^{n} v=y$ and $\lim _{n \rightarrow \infty} g^{n} z=w$.

Proof. Let $x_{0}, y_{0}, z_{0} \in X$ be arbitrary. Choose $x_{1}, y_{1}, z_{1} \in$ $X$ such that $g x_{1} \in S\left(x_{0}, y_{0}, z_{0}\right), g y_{1} \in S\left(y_{0}, z_{0}, x_{0}\right)$ and $g z_{1} \in S\left(z_{0}, x_{0}, y_{0}\right)$. Choose $x_{2}, y_{2}, z_{2} \in X$ such that $g x_{2} \in$ $T\left(x_{1}, y_{1}, z_{1}\right), g y_{2} \in T\left(y_{1}, z_{1}, x_{1}\right)$, and $g z_{2} \in T\left(z_{1}, x_{1}, y_{1}\right)$. This can be done because $S(X \times X \times X) \cup T(X \times X \times X) \subseteq g(X)$. If

$$
a_{1}=a_{2}=a_{3}=a_{4}=a_{5}=a_{6}=a_{7}=0,
$$

then

$$
\begin{aligned}
& d\left(g x_{1}, T\left(x_{1}, y_{1}, z_{1}\right)\right) \leq H\left(S\left(x_{0}, y_{0}, z_{0}\right), T\left(x_{1}, y_{1}, z_{1}\right)\right)=0 \\
& d\left(g x_{2}, S\left(x_{2}, y_{2}, z_{2}\right)\right) \leq H\left(T\left(x_{1}, y_{1}, z_{1}\right), S\left(x_{2}, y_{2}, z_{2}\right)\right)=0 .
\end{aligned}
$$

Imply that

$$
d\left(g x_{1}, T\left(x_{1}, y_{1}, z_{1}\right)\right)=0, \quad d\left(g x_{2}, S\left(x_{2}, y_{2}, z_{2}\right)\right)=0 .
$$

As $T\left(x_{1}, y_{1}, z_{1}\right)$ and $S\left(x_{2}, y_{2}, z_{2}\right)$ are closed,

$$
g x_{1} \in T\left(x_{1}, y_{1}, z_{1}\right), \quad g x_{2} \in S\left(x_{2}, y_{2}, z_{2}\right) \text {. }
$$

Similarly

$$
\begin{array}{ll}
g y_{1} \in T\left(y_{1}, z_{1}, x_{1}\right), & g y_{2} \in S\left(y_{2}, z_{2}, x_{2}\right), \\
g z_{1} \in T\left(z_{1}, x_{1}, y_{1}\right), & g z_{2} \in S\left(z_{2}, x_{2}, y_{2}\right) .
\end{array}
$$

Hence $\left(x_{1}, y_{1}, z_{1}\right)$ and $\left(x_{2}, y_{2}, z_{2}\right)$ are tripled coincidence points of pairs $(T, g)$ and $(S, g)$, respectively. Now assume that $a_{i}>0$, for some $i=1,2, \ldots, 7$ which gives that $h>0$; therefore, there exist

$$
\begin{array}{ll}
t_{1} \in T\left(x_{1}, y_{1}, z_{1}\right), & t_{2} \in T\left(y_{1}, z_{1}, x_{1}\right), \\
t_{3} \in T\left(z_{1}, x_{1}, y_{1}\right), & t_{4} \in S\left(x_{2}, y_{2}, z_{2}\right), \\
t_{5} \in S\left(y_{2}, z_{2}, x_{2}\right), & t_{6} \in S\left(z_{2}, x_{2}, y_{2}\right),
\end{array}
$$

such that

$$
\begin{aligned}
& d\left(g x_{1}, t_{1}\right) \leq H\left(S\left(x_{0}, y_{0}, z_{0}\right), T\left(x_{1}, y_{1}, z_{1}\right)\right)+\frac{h}{6}, \\
& d\left(g y_{1}, t_{2}\right) \leq H\left(S\left(y_{0}, z_{0}, x_{0}\right), T\left(y_{1}, z_{1}, x_{1}\right)\right)+\frac{h}{6}, \\
& d\left(g z_{1}, t_{3}\right) \leq H\left(S\left(z_{0}, x_{0}, y_{0}\right), T\left(z_{1}, x_{1}, y_{1}\right)\right)+\frac{h}{6}, \\
& d\left(g x_{2}, t_{4}\right) \leq H\left(T\left(x_{1}, y_{1}, z_{1}\right), S\left(x_{2}, y_{2}, z_{2}\right)\right)+\frac{h}{6}, \\
& d\left(g y_{2}, t_{5}\right) \leq H\left(T\left(y_{1}, z_{1}, x_{1}\right), S\left(y_{2}, z_{2}, x_{2}\right)\right)+\frac{h}{6}, \\
& d\left(g z_{2}, t_{6}\right) \leq H\left(T\left(z_{1}, x_{1}, y_{1}\right), S\left(z_{2}, x_{2}, y_{2}\right)\right)+\frac{h}{6} .
\end{aligned}
$$


Since $S(X \times X \times X) \cup T(X \times X \times X) \subseteq g(X)$, there exist $x_{2}, y_{2}$, $z_{2}, x_{3}, y_{3}$, and $z_{3}$ in $X$ such that $t_{1}=g x_{2}, t_{2}=g y_{2}, t_{3}=g z_{2}$, $t_{4}=g x_{3}, t_{5}=g y_{3}$, and $t_{6}=g z_{3}$. Thus

$$
\begin{aligned}
& d\left(g x_{1}, g x_{2}\right) \leq H\left(S\left(x_{0}, y_{0}, z_{0}\right), T\left(x_{1}, y_{1}, z_{1}\right)\right)+\frac{h}{6}, \\
& d\left(g y_{1}, g y_{2}\right) \leq H\left(S\left(y_{0}, z_{0}, x_{0}\right), T\left(y_{1}, z_{1}, x_{1}\right)\right)+\frac{h}{6}, \\
& d\left(g z_{1}, g z_{2}\right) \leq H\left(S\left(z_{0}, x_{0}, y_{0}\right), T\left(z_{1}, x_{1}, y_{1}\right)\right)+\frac{h}{6}, \\
& d\left(g x_{2}, g x_{3}\right) \leq H\left(T\left(x_{1}, y_{1}, z_{1}\right), S\left(x_{2}, y_{2}, z_{2}\right)\right)+\frac{h}{6}, \\
& d\left(g y_{2}, g y_{3}\right) \leq H\left(T\left(y_{1}, z_{1}, x_{1}\right), S\left(y_{2}, z_{2}, x_{2}\right)\right)+\frac{h}{6}, \\
& d\left(g z_{2}, g z_{3}\right) \leq H\left(T\left(z_{1}, x_{1}, y_{1}\right), S\left(z_{2}, x_{2}, y_{2}\right)\right)+\frac{h}{6} .
\end{aligned}
$$

Continuing this process, we obtain three sequences $\left\{x_{n}\right\},\left\{y_{n}\right\}$, and $\left\{z_{n}\right\}$ in $X$ such that

$$
\begin{gathered}
g x_{2 n+1} \in S\left(x_{2 n}, y_{2 n}, z_{2 n}\right), \quad g y_{2 n+1} \in S\left(y_{2 n}, z_{2 n}, x_{2 n}\right), \\
g z_{2 n+1} \in S\left(z_{2 n}, x_{2 n}, y_{2 n}\right), \\
g x_{2 n+2} \in T\left(x_{2 n+1}, y_{2 n+1}, z_{2 n+1}\right), \\
g y_{2 n+2} \in T\left(y_{2 n+1}, z_{2 n+1}, x_{2 n+1}\right), \\
g z_{2 n+2} \in T\left(z_{2 n+1}, x_{2 n+1}, y_{2 n+1}\right),
\end{gathered}
$$

with

$$
\begin{gathered}
d\left(g x_{2 n+1}, g x_{2 n+2}\right) \\
\leq H\left(S\left(x_{2 n}, y_{2 n}, z_{2 n}\right), T\left(x_{2 n+1}, y_{2 n+1}, z_{2 n+1}\right)\right)+\frac{h^{n}}{6} \\
d\left(g y_{2 n+1}, g y_{2 n+2}\right) \\
\quad \leq H\left(S\left(y_{2 n}, z_{2 n}, x_{2 n}\right), T\left(y_{2 n+1}, z_{2 n+1}, x_{2 n+1}\right)\right)+\frac{h^{n}}{6} \\
d\left(g z_{2 n+1}, g z_{2 n+2}\right) \\
\quad \leq H\left(S\left(z_{2 n}, x_{2 n}, y_{2 n}\right), T\left(z_{2 n+1}, x_{2 n+1}, y_{2 n+1}\right)\right)+\frac{h^{n}}{6} \\
d\left(g x_{2 n+2}, g x_{2 n+3}\right) \\
\leq H\left(T\left(x_{2 n+1}, y_{2 n+1}, z_{2 n+1}\right), S\left(x_{2 n+2}, y_{2 n+2}, z_{2 n+2}\right)\right)+\frac{h^{n}}{6} \\
d\left(g y_{2 n+2}, g y_{2 n+3}\right) \\
\quad \leq H\left(T\left(y_{2 n+1}, z_{2 n+1}, x_{2 n+1}\right), S\left(y_{2 n+2}, z_{2 n+2}, x_{2 n+2}\right)\right)+\frac{h^{n}}{6} \\
d\left(g z_{2 n+2}, g z_{2 n+3}\right) \\
\leq H\left(T\left(z_{2 n+1}, x_{2 n+1}, y_{2 n+1}\right), S\left(z_{2 n+2}, x_{2 n+2}, y_{2 n+2}\right)\right)+\frac{h^{n}}{6} .
\end{gathered}
$$

By (4),

$$
\begin{aligned}
& d\left(g x_{2 n+1}, g x_{2 n+2}\right) \\
& \leq H\left(S\left(x_{2 n}, y_{2 n}, z_{2 n}\right), T\left(x_{2 n+1}, y_{2 n+1}, z_{2 n+1}\right)\right)+\frac{h^{n}}{6} \\
& \leq a_{1} d\left(g x_{2 n}, g x_{2 n+1}\right)+a_{2} d\left(g y_{2 n}, g y_{2 n+1}\right) \\
&+a_{3} d\left(g z_{2 n}, g z_{2 n+1}\right)+a_{4} d\left(S\left(x_{2 n}, y_{2 n}, z_{2 n}\right), g x_{2 n}\right) \\
&+a_{5} d\left(T\left(x_{2 n+1}, y_{2 n+1}, z_{2 n+1}\right), g x_{2 n+1}\right) \\
&+a_{6} d\left(S\left(x_{2 n}, y_{2 n}, z_{2 n}\right), g x_{2 n+1}\right) \\
&+a_{7} d\left(T\left(x_{2 n+1}, y_{2 n+1}, z_{2 n+1}\right), g x_{2 n}\right)+\frac{h^{n}}{6} \\
& \leq a_{1} d\left(g x_{2 n}, g x_{2 n+1}\right)+a_{2} d\left(g y_{2 n}, g y_{2 n+1}\right) \\
&+a_{3} d\left(g z_{2 n}, g z_{2 n+1}\right)+a_{4} d\left(g x_{2 n+1}, g x_{2 n}\right) \\
&+a_{5} d\left(g x_{2 n+2}, g x_{2 n+1}\right)+a_{6} d\left(g x_{2 n+1}, g x_{2 n+1}\right) \\
&+a_{7} d\left(g x_{2 n+2}, g x_{2 n}\right)+\frac{h^{n}}{6},
\end{aligned}
$$

which further gives

$$
\begin{aligned}
& \left(1-a_{5}-a_{7}\right) d\left(g x_{2 n+1}, g x_{2 n+2}\right) \\
& \leq\left(a_{1}+a_{4}+a_{7}\right) d\left(g x_{2 n}, g x_{2 n+1}\right) \\
& \quad+a_{2} d\left(g y_{2 n}, g y_{2 n+1}\right)+a_{3} d\left(g z_{2 n}, g z_{2 n+1}\right)+\frac{h^{n}}{6} .
\end{aligned}
$$

Similarly it can be shown that

$$
\begin{aligned}
(1- & \left.a_{5}-a_{7}\right) d\left(g y_{2 n+1}, g y_{2 n+2}\right) \\
\leq & \left(a_{1}+a_{4}+a_{7}\right) d\left(g y_{2 n}, g y_{2 n+1}\right) \\
\quad & +a_{2} d\left(g z_{2 n}, g z_{2 n+1}\right)+a_{3} d\left(g x_{2 n}, g x_{2 n+1}\right)+\frac{h^{n}}{6}, \\
(1- & \left.a_{5}-a_{7}\right) d\left(g z_{2 n+1}, g z_{2 n+2}\right) \\
\leq & \left(a_{1}+a_{4}+a_{7}\right) d\left(g z_{2 n}, g z_{2 n+1}\right) \\
& +a_{2} d\left(g x_{2 n}, g x_{2 n+1}\right)+a_{3} d\left(g y_{2 n}, g y_{2 n+1}\right)+\frac{h^{n}}{6} .
\end{aligned}
$$


Again

$$
\begin{aligned}
& d\left(g x_{2 n+2}, g x_{2 n+1}\right) \\
& \leq H\left(T\left(x_{2 n+1}, y_{2 n+1}, z_{2 n+1}\right), S\left(x_{2 n}, y_{2 n}, z_{2 n}\right)\right)+\frac{h^{n}}{6} \\
& \leq a_{1} d\left(g x_{2 n+1}, g x_{2 n}\right)+a_{2} d\left(g y_{2 n+1}, g y_{2 n}\right) \\
&+a_{3} d\left(g z_{2 n+1}, g z_{2 n}\right) \\
&+a_{4} d\left(T\left(x_{2 n+1}, y_{2 n+1}, z_{2 n+1}\right), g x_{2 n+1}\right) \\
&+a_{5} d\left(S\left(x_{2 n}, y_{2 n}, z_{2 n}\right), g x_{2 n}\right) \\
&+a_{6} d\left(T\left(x_{2 n+1}, y_{2 n+1}, z_{2 n+1}\right), g x_{2 n}\right) \\
&+a_{7} d\left(S\left(x_{2 n}, y_{2 n}, z_{2 n}\right), g x_{2 n+1}\right)+\frac{h^{n}}{6} \\
& \leq a_{1} d\left(g x_{2 n+1}, g x_{2 n}\right)+a_{2} d\left(g y_{2 n+1}, g y_{2 n}\right) \\
&+a_{3} d\left(g z_{2 n+1}, g z_{2 n}\right)+a_{4} d\left(g x_{2 n+2}, g x_{2 n+1}\right) \\
&+a_{5} d\left(g x_{2 n+1}, g x_{2 n}\right)+a_{6} d\left(g x_{2 n+2}, g x_{2 n}\right) \\
&+a_{7} d\left(g x_{2 n+1}, g x_{2 n+1}\right)+\frac{h^{n}}{6},
\end{aligned}
$$

which implies

$$
\begin{aligned}
& \left(1-a_{4}-a_{6}\right) d\left(g x_{2 n+2}, g x_{2 n+1}\right) \\
& \leq\left(a_{1}+a_{5}+a_{6}\right) d\left(g x_{2 n+1}, g x_{2 n}\right) \\
& \quad+a_{2} d\left(g y_{2 n+1}, g y_{2 n}\right)+a_{3} d\left(g z_{2 n+1}, g z_{2 n}\right)+\frac{h^{n}}{6} .
\end{aligned}
$$

Similarly, it can be shown that

$$
\begin{aligned}
(1- & \left.a_{4}-a_{6}\right) d\left(g y_{2 n+2}, g y_{2 n+1}\right) \\
\leq & \left(a_{1}+a_{5}+a_{6}\right) d\left(g y_{2 n+1}, g y_{2 n}\right) \\
& \quad+a_{2} d\left(g z_{2 n+1}, g z_{2 n}\right)+a_{3} d\left(g x_{2 n+1}, g x_{2 n}\right)+\frac{h^{n}}{6}, \\
(1- & \left.a_{4}-a_{6}\right) d\left(g z_{2 n+2}, g z_{2 n+1}\right) \\
\leq & \left(a_{1}+a_{5}+a_{6}\right) d\left(g z_{2 n+1}, g z_{2 n}\right) \\
& +a_{2} d\left(g x_{2 n+1}, g x_{2 n}\right)+a_{3} d\left(g y_{2 n+1}, g y_{2 n}\right)+\frac{h^{n}}{6} .
\end{aligned}
$$

Let

$$
\begin{aligned}
\delta_{2 n+1}= & d\left(g x_{2 n+1}, g x_{2 n+2}\right)+d\left(g y_{2 n+1}, g y_{2 n+2}\right) \\
& +d\left(g z_{2 n+1}, g z_{2 n+2}\right) .
\end{aligned}
$$

From (17) and (18), we get

$$
\left(1-a_{5}-a_{7}\right) \delta_{2 n+1} \leq\left(a_{1}+a_{2}+a_{3}+a_{4}+a_{7}\right) \delta_{2 n}+\frac{h^{n}}{2} .
$$

From (20) and (21) we get

$$
\left(1-a_{4}-a_{6}\right) \delta_{2 n+1} \leq\left(a_{1}+a_{2}+a_{3}+a_{5}+a_{6}\right) \delta_{2 n}+\frac{h^{n}}{2} .
$$

Adding (23) and (24), we obtain

$$
\begin{aligned}
(2 & \left.-a_{4}-a_{5}-a_{6}-a_{7}\right) \delta_{2 n+1} \\
& \leq\left(2 a_{1}+2 a_{2}+2 a_{3}+a_{4}+a_{5}+a_{6}+a_{7}\right) \delta_{2 n}+h^{n} .
\end{aligned}
$$

Since by inequality (5), we get

$$
a_{1}+a_{2}+a_{3}+a_{4}+a_{5}+a_{6}+a_{7} \leq h<1 .
$$

Hence

$$
\begin{aligned}
2\left(a_{1}+\right. & \left.a_{2}+a_{3}\right)+a_{4}+a_{5}+a_{6}+a_{7} \\
= & 2\left(a_{1}+a_{2}+a_{3}+a_{4}+a_{5}+a_{6}+a_{7}\right) \\
& -\left(a_{4}+a_{5}+a_{6}+a_{7}\right) \\
\leq & 2 h-\left(a_{4}+a_{5}+a_{6}+a_{7}\right) \\
\leq & 2 h-h\left(a_{4}+a_{5}+a_{6}+a_{7}\right) \\
\leq & h\left(2-a_{4}-a_{5}-a_{6}-a_{7}\right) .
\end{aligned}
$$

Then from (27), we get

$$
\begin{aligned}
& \left(2-a_{4}-a_{5}-a_{6}-a_{7}\right) \delta_{2 n+1} \\
& \quad \leq h\left(2-a_{4}-a_{5}-a_{6}-a_{7}\right) \delta_{2 n}+h^{n} .
\end{aligned}
$$

As $1 /\left(2-a_{4}-a_{5}-a_{6}-a_{7}\right)<1$,

$$
\delta_{2 n+1} \leq h \delta_{2 n}+h^{n} \text {. }
$$

By the similar process as above, we can show that

$$
\delta_{2 n} \leq h \delta_{2 n-1}+h^{n}
$$

Thus we have

$$
\begin{aligned}
\delta_{2 n+1} & \leq h\left(h \delta_{2 n-1}+h^{n}\right)+h^{n} \\
& =h^{2} \delta_{2 n-1}+h^{n+1}+h^{n} \\
& \leq h^{2}\left(h \delta_{2 n-2}+h^{n-1}\right)+h^{n+1}+h^{n} \\
& =h^{3} \delta_{2 n-2}+2 h^{n+1}+h^{n} .
\end{aligned}
$$

Continuing this process, we obtain

$$
\begin{aligned}
\delta_{2 n+1} & \leq h^{n+1} \delta_{2}+n h^{n+1}+h^{n} \\
& \leq h^{n+1}\left(h \delta_{1}+h\right)+n h^{n+1}+h^{n} \\
& =h^{n+2} \delta_{1}+h^{n+2}+n h^{n+1}+h^{n} \\
& \leq h^{n+2}\left(h \delta_{0}+1\right)+h^{n+2}+n h^{n+1}+h^{n} \\
& =h^{n+3} \delta_{0}+2 h^{n+2}+n h^{n+1}+h^{n} \\
& \leq h^{n} \delta_{0}+(n+3) h^{n} .
\end{aligned}
$$


Similarly

$$
\delta_{2 n} \leq h\left(h \delta_{2 n-2}+h^{n-1}\right)+h^{n}=h^{2} \delta_{2 n-2}+2 h^{n} .
$$

Continuation of this process implies that

$$
\begin{aligned}
\delta_{2 n} & \leq h^{2 n-1} \delta_{1}+(2 n-1) h^{n} \\
& \leq h^{2 n-1}\left(h \delta_{0}+1\right)+(2 n-1) h^{n} \\
& =h^{2 n} \delta_{0}+h^{2 n-1}+(2 n-1) h^{n} \\
& \leq h^{n} \delta_{0}+2 n h^{n} .
\end{aligned}
$$

By (32) and (34), we have

$$
\begin{aligned}
\delta_{n} & \leq h^{n+3} \delta_{0}+2 h^{n+2}+(n+1) h^{n} \\
& \leq h^{n} \delta_{0}+2 n h^{n} \quad \forall n \geq 3 .
\end{aligned}
$$

That is,

$$
\delta_{n} \leq h^{n} \delta_{0}+2 n h^{n}
$$

holds true for all $n \geq 3$, where

$$
\delta_{n}=d\left(g x_{n}, g x_{n+1}\right)+d\left(g y_{n}, g y_{n+1}\right)+d\left(g z_{n}, g z_{n+1}\right) .
$$

Now for every $m, n \in N$ with $m>n \geq 3$, we have

$$
\begin{aligned}
& d\left(g x_{n}, g x_{m+n}\right)+d\left(g y_{n}, g y_{m+n}\right)+d\left(g z_{n}, g z_{m+n}\right) \\
& \leq d\left(g x_{n}, g x_{n+1}\right)+d\left(g x_{n+1}, g x_{n+2}\right) \\
&+\cdots+d\left(g x_{n+m-1}, g x_{n+m}\right)+d\left(g y_{n}, g y_{n+1}\right) \\
&+d\left(g y_{n+1}, g y_{n+2}\right)+\cdots+d\left(g y_{n+m-1}, g y_{n+m}\right) \\
&+d\left(g z_{n}, g z_{n+1}\right)+d\left(g z_{n+1}, g z_{n+2}\right) \\
&+\cdots+d\left(g z_{n+m-1}, g z_{n+m}\right) \\
& \leq\left(h^{n} \delta_{0}+2 n h^{n}\right)+\left(h^{n+1} \delta_{0}+2(n+1) h^{n+1}\right) \\
&+\cdots+\left(h^{n+m-1} \delta_{0}+2(n+m-1) h^{n+m-1}\right) \\
& \leq\left(\sum_{i=n}^{m+n-1} h^{i} \delta_{0}+2 \sum_{i=n}^{m+n-1} i h^{i}\right) .
\end{aligned}
$$

Since $h<1$, we conclude that $\left\{g x_{n}\right\},\left\{g y_{n}\right\}$, and $\left\{g z_{n}\right\}$ are Cauchy sequences in $g(X)$. By completeness of $g(X)$, there exists $x, y, z \in X$ such that $g x_{n} \rightarrow g x, g y_{n} \rightarrow g y$, and $g z_{n} \rightarrow g z$. Then from (4), we get

$$
\begin{aligned}
d(T & (x, y, z), g x) \leq d\left(T(x, y, z), g x_{2 n+1}\right)+d\left(g x_{2 n+1}, g x\right) \\
d( & (x, y, z), g x) \\
\leq & H\left(T(x, y, z), S\left(x_{2 n}, y_{2 n}, z_{2 n}\right)\right)+d\left(g x_{2 n+1}, g x\right) \\
\leq & a_{1} d\left(g x, g x_{2 n}\right)+a_{2} d\left(g y, g y_{2 n}\right)+a_{3} d\left(g z, g z_{2 n}\right) \\
& +a_{4} d(T(x, y, z), g x)+a_{5} d\left(S\left(x_{2 n}, y_{2 n}, z_{2 n}\right), g x_{2 n}\right) \\
& +a_{6} d\left(T(x, y, z), g x_{2 n}\right)+a_{7} d\left(S\left(x_{2 n}, y_{2 n}, z_{2 n}\right), g x\right) \\
& +d\left(g x_{2 n+1}, g x\right) \\
\leq & a_{1} d\left(g x, g x_{2 n}\right)+a_{2} d\left(g y, g y_{2 n}\right)+a_{3} d\left(g z, g z_{2 n}\right) \\
& +a_{4} d(T(x, y, z), g x)+a_{5} d\left(g x_{2 n+1}, g x_{2 n}\right) \\
& +a_{6} d\left(T(x, y, z), g x_{2 n}\right)+a_{7} d\left(g x_{2 n+1}, g x\right) \\
& +d\left(g x_{2 n+1}, g x\right) .
\end{aligned}
$$

On taking limits as $n \rightarrow \infty$, we get

$$
d(T(x, y, z), g x) \leq\left(a_{4}+a_{6}\right) d(T(x, y, z), g x),
$$

which implies that

$$
d(T(x, y, z), g x)=0 .
$$

And hence $g x \in T(x, y, z)$. Similarly $g y \in T(y, z, x)$, $g z \in T(z, x, y)$. And $g x \in S(x, y, z), g y \in S(y, z, x), g z \in$ $S(z, x, y)$. Thus $(x, y, z)$ is a tripled coincidence point of $(S, g)$ and $(T, g)$. Suppose that (i) holds; then, for some $(x, y, z) \in$ $\Upsilon(g, S)$ and $\Upsilon(g, T)$, we have $g x \in S(x, y, z), g y \in S(y, z, x)$, $g z \in S(z, x, y)$, and $g x \in T(x, y, z), g y \in T(y, z, x), g z \in$ $S(z, x, y)$. Since $(S, g)$ and $(T, g)$ are $w$-compatible, we have

$$
\begin{aligned}
& g(S(x, y, z)) \subseteq S(g x, g y, g z), \\
& g(T(x, y, z)) \subseteq T(g x, g y, g z),
\end{aligned}
$$

for $(x, y, z) \in \Upsilon(g, S)$ and $\Upsilon(g, T)$. Since $g x \in S(x, y, z), g y \in$ $S(y, z, x)$ and $g z \in S(z, x, y)$. So $g^{2} x \in S(g x, g y, g z), g^{2} y \in$ $S(g y, g z, g x)$, and $g^{2} z \in S(g z, g x, g y)(\Rightarrow(g x, g y, g z) \epsilon$ $\Upsilon(g, S))$. Similarly $g^{2} x \in T(g x, g y, g z), g^{2} y \in T(g y, g z, g x)$, and $g^{2} z \in T(g z, g x, g y)(\Rightarrow(g x, g y, g z) \in \Upsilon(g, T))$. Continuing in this way, we get

$$
\begin{aligned}
& g^{n} x \in S\left(g^{n-1} x, g^{n-1} y, g^{n-1} z\right), \\
& g^{n} y \in S\left(g^{n-1} y, g^{n-1} z, g^{n-1} x\right), \\
& g^{n} z \in S\left(g^{n-1} z, g^{n-1} x, g^{n-1} y\right),
\end{aligned}
$$

which implies

$$
\begin{aligned}
& \left(g^{n-1} x, g^{n-1} y, g^{n-1} z\right) \in \Upsilon(g, S), \\
& \left(g^{n-1} x, g^{n-1} y, g^{n-1} z\right) \in \Upsilon(g, T)
\end{aligned}
$$


for all $n \geq 1$ and

$$
\begin{aligned}
& g^{n} x \in S\left(g^{n-1} x, g^{n-1} y, g^{n-1} z\right), \\
& g^{n} x \in T\left(g^{n-1} x, g^{n-1} y, g^{n-1} z\right) .
\end{aligned}
$$

Since $\lim _{n \rightarrow \infty} g^{n} x=u, \lim _{n \rightarrow \infty} g^{n} y=v$ and $\lim _{n \rightarrow \infty} g^{n} z=$ $w$ for $(x, y, z) \in \Upsilon(S, g)$ and $\Upsilon(T, g)$ and $g$ is continuous at $u$, $v$ and $w$, so we have $u=g u, v=g v$, and $w=g w$. Now using (4), we get

$$
\begin{aligned}
& d(g u, S(u, v, w)) \leq d\left(g u, g^{n} x\right)+d\left(g^{n} x, S(u, v, w)\right) \\
& d(g u, S(u, v, w)) \leq d\left(g u, g^{n} x\right)+H\left(T\left(g^{n-1} x, g^{n-1} y, g^{n-1} z\right), S(u, v, w)\right) \\
& d(g u, S(u, v, w)) \\
& \leq d\left(g u, g^{n} x\right)+a_{1} d\left(g^{n} x, g u\right)+a_{2} d\left(g^{n} y, g v\right) \\
&+a_{3} d\left(g^{n} z, g w\right)+a_{4} d\left(T\left(g^{n-1} x, g^{n-1} y, g^{n-1} z\right), g^{n} x\right) \\
&+a_{5} d(S(u, v, z), g u) \\
&+a_{6} d\left(T\left(g^{n-1} x, g^{n-1} y, g^{n-1} z\right), g u\right) \\
&+a_{7} d\left(S(u, v, w), g^{n} x\right) \\
& \leq d\left(g u, g^{n} x\right)+a_{1} d\left(g^{n} x, g u\right) \\
&+a_{2} d\left(g^{n} y, g v\right)+a_{3} d\left(g^{n} z, g w\right) \\
&+a_{4} d\left(g^{n} x, g^{n} x\right)+a_{5} d(S(u, v, z), g u) \\
&+a_{6} d\left(g^{n} x, g u\right)+a_{7} d\left(S(u, v, w), g^{n} x\right)
\end{aligned}
$$

On taking limits as $n \rightarrow \infty$, we get

$$
d(g u, S(u, v, w)) \leq\left(a_{5}+a_{7}\right) d(g u, S(u, v, w)),
$$

which implies that

$$
d(g u, S(u, v, w))=0,
$$

and hence

$$
g u \in S(u, v, w)
$$

Similarly,

$$
g v \in S(v, w, u), \quad g w \in S(w, u, v) .
$$

Consequently,

$$
\begin{gathered}
u=g u \in S(u, v, w), \quad v=g v \in S(v, w, u), \\
w=g w \in S(w, u, v) .
\end{gathered}
$$

Similarly,

$$
\begin{gathered}
u=g u \in T(u, v, w), \quad v=g v \in T(v, w, u) \\
w=g w \in T(w, u, v) .
\end{gathered}
$$

Hence $(u, v, w)$ is a tripled fixed point of $(S, g)$ and $(T, g)$. Now suppose that (ii) holds. Since $g$ is $S, T$-idempotent for some $(x, y, z) \in \Upsilon(g, S)$ and $(x, y, z) \in \Upsilon(g, T)$, we have $g^{2} x \in$ $S(g x, g y, g z), g^{2} y \in S(g y, g z, g x), g^{2} z \in S(g z, g x, g y)$, and $g^{2} x \in T(g x, g y, g z), g^{2} y \in T(g y, g z, g x), g^{2} z \epsilon$ $T(g z, g x, g y)$. Since we have $g^{2} x=g x, g^{2} y=g y$ and $g^{2} z=g z$. So

$$
\begin{gathered}
g x=g^{2} x \in S(g x, g y, g z), \quad g y=g^{2} y \in S(g y, g z, g x), \\
g z=g^{2} z \in S(g z, g x, g y), \\
g x=g^{2} x \in T(g x, g y, g z), \quad g y=g^{2} y \in T(g y, g z, g x), \\
g z=g^{2} z \in T(g z, g x, g y) .
\end{gathered}
$$

Hence $(g x, g y, g z)$ is a tripled common fixed point of $(S, g)$ and $(T, g)$. Now suppose that (iii) holds. For some $(x, y, z) \in$ $\Upsilon(g, S)$ and $\Upsilon(g, T)$, we get

$$
\begin{array}{ll}
g x \in S(x, y, z), & g y \in S(y, z, x), \\
g z \in S(z, x, y), & g x \in T(x, y, z), \\
g y \in T(y, z, x), & g z \in T(z, x, y) .
\end{array}
$$

Since $g$ is continuous at $x$ and $y$, we get $g x=x, g y=y$, and $g z=z$. Thus

$$
\begin{gathered}
x=g x \in S(x, y, z), \quad y=g y \in S(y, z, x), \\
z=g z \in S(z, x, y), \\
x=g x \in T(x, y, z), \quad y=g y \in T(y, z, x), \\
z=g z \in T(z, x, y) .
\end{gathered}
$$

This implies that $(x, y, z)$ is a tripled common fixed point of $(S, g)$ and $(T, g)$.

If in Theorem 7, $S=T, g=I$ (the identity mapping), then we have the following result.

Corollary 8. Let $(X, d)$ be a metric space and $T: X \times X \times$ $X \rightarrow C B(X)$ such that

$$
\begin{aligned}
H( & (x, y, z), T(u, v, w)) \\
\leq & a_{1} d(x, u)+a_{2} d(y, v)+a_{3} d(z, w) \\
& +a_{4} d(T(x, y, z), x)+a_{5} d(T(u, v, w), u) \\
& +a_{6} d(T(x, y, z), u)+a_{7} d(T(u, v, z), x),
\end{aligned}
$$

for all $x, y, z, u, v, w \in X$, where $a_{i}=a_{i}(x, y, z, u, v, w), i=$ $1,2, \ldots, 7$, are nonnegative real satisfy (5). Then $T$ has a fixed point.

Corollary 9. Let $(X, d)$ be a metric space and $S, T: X \times X \times$ $X \rightarrow C B(X)$ and $g: X \rightarrow X$ be mappings such that

$$
\begin{aligned}
& H(S(x, y, z), T(u, v, w)) \\
& \quad \leq \frac{k}{2}[d(g x, g u)+d(g y, g v)+d(g z, g w)]
\end{aligned}
$$


for all $x, y, z, u, v, w \in X$, where $k \in[0,1)$. If $S(X \times X \times X) \cup$ $T(X \times X \times X) \subseteq g(X)$ and $g(X)$ is complete subset of $X$. Then $(S, g)$ and $(T, g)$ have tripled coincidence point. Moreover $(S, g)$ and $(T, g)$ have tripled common fixed point if anyone of the conditions (i)-(iii) of Theorem 7 holds.

Theorem 10. Let $(X, d)$ be a metric space and $S, T: X \times X \times$ $X \rightarrow C B(X)$ and $g: X \rightarrow X$ be mappings such that

$$
\begin{aligned}
& H(S(x, y, z), T(u, v, w)) \\
& \leq h \max \{d(g x, g u), d(g y, g v), d(g z, g w), \\
& d(S(x, y, z), g x), d(T(u, v, w), g u), \\
&\left.\frac{d(S(x, y, z), g x)+d(T(u, v, z), g u)}{2}\right\}
\end{aligned}
$$

for all $x, y, z, u, v, w \in X$, where $h \in[0,1)$. If $S(X \times X \times$ $X) \cup T(X \times X \times X) \subseteq g(X)$ and $g(X)$ is complete subset of $X$. Then $(S, g)$ and $(T, g)$ have tripled coincidence point. Moreover $(S, g)$ and $(T, g)$ have tripled common fixed point if one of the conditions (i)-(iii) of Theorem 7 holds.

Proof. Let $x_{0}, y_{0}, z_{0} \in X$ be arbitrary. Choose $x_{1}, y_{1}, z_{1} \in$ $X$ such that $g x_{1} \in S\left(x_{0}, y_{0}, z_{0}\right), g y_{1} \in S\left(y_{0}, z_{0}, x_{0}\right)$ and $g z_{1} \in S\left(z_{0}, x_{0}, y_{0}\right)$. Note that $T\left(x_{1}, y_{1}, z_{1}\right), T\left(y_{1}, z_{1}, x_{1}\right)$ and $T\left(z_{1}, x_{1}, y_{1}\right)$ are well defined. Choose $x_{2}, y_{2}, z_{2} \in X$ such that $g x_{2} \in T\left(x_{1}, y_{1}, z_{1}\right), g y_{2} \in T\left(y_{1}, z_{1}, x_{1}\right)$, and $g z_{2} \in$ $T\left(z_{1}, x_{1}, y_{1}\right)$. If $h=0$, then following similar arguments to those given in Theorem 7 , we obtain that $\left(x_{1}, y_{1}, z_{1}\right)$ and $\left(x_{2}, y_{2}, z_{2}\right)$ are tripled coincidence point $(T, g)$ and $(S, g)$, respectively. Now assume that $h>0$, set $k=1 / \sqrt{h}$. Then $k>1$, so there exists

$$
\begin{array}{ll}
t_{1} \in T\left(x_{1}, y_{1}, z_{1}\right), & t_{2} \in T\left(y_{1}, z_{1}, x_{1}\right), \\
t_{3} \in T\left(z_{1}, x_{1}, y_{1}\right), & t_{4} \in S\left(x_{2}, y_{2}, z_{2}\right), \\
t_{5} \in S\left(y_{2}, z_{2}, x_{2}\right), & t_{6} \in S\left(z_{2}, x_{2}, y_{2}\right),
\end{array}
$$

such that

$$
\begin{aligned}
& d\left(g x_{1}, t_{1}\right) \leq k H\left(S\left(x_{0}, y_{0}, z_{0}\right), T\left(x_{1}, y_{1}, z_{1}\right)\right), \\
& d\left(g y_{1}, t_{2}\right) \leq k H\left(S\left(y_{0}, z_{0}, x_{0}\right), T\left(y_{1}, z_{1}, x_{1}\right)\right), \\
& d\left(g z_{1}, t_{3}\right) \leq k H\left(S\left(z_{0}, x_{0}, y_{0}\right), T\left(z_{1}, x_{1}, y_{1}\right)\right), \\
& d\left(g x_{2}, t_{4}\right) \leq k H\left(T\left(x_{1}, y_{1}, z_{1}\right), S\left(x_{2}, y_{2}, z_{2}\right)\right),
\end{aligned}
$$

$$
\begin{aligned}
& d\left(g y_{2}, t_{5}\right) \leq k H\left(T\left(y_{1}, z_{1}, x_{1}\right), S\left(y_{2}, z_{2}, x_{2}\right)\right), \\
& d\left(g z_{2}, t_{6}\right) \leq k H\left(T\left(z_{1}, x_{1}, y_{1}\right), S\left(z_{2}, x_{2}, y_{2}\right)\right) .
\end{aligned}
$$

Since $S(X \times X \times X) \cup T(X \times X \times X) \subseteq g(X)$, there exist $x_{2}, y_{2}$, $z_{2}, x_{3}, y_{3}$, and $z_{4}$ in $X$ such that $t_{1}=g x_{2}, t_{2}=g y_{2}, t_{3}=g z_{2}$, $t_{4}=g x_{3}, t_{5}=g y_{3}$, and $t_{6}=g z_{3}$. Thus

$$
\begin{aligned}
& d\left(g x_{1}, g x_{2}\right) \leq k H\left(S\left(x_{0}, y_{0}, z_{0}\right), T\left(x_{1}, y_{1}, z_{0}\right)\right), \\
& d\left(g y_{1}, g y_{2}\right) \leq k H\left(S\left(y_{0}, z_{0}, x_{0}\right), T\left(y_{1}, z_{1}, x_{1}\right)\right), \\
& d\left(g z_{1}, g z_{2}\right) \leq k H\left(S\left(z_{0}, x_{0}, y_{0}\right), T\left(z_{1}, x_{1}, y_{1}\right)\right), \\
& d\left(g x_{2}, g x_{3}\right) \leq k H\left(T\left(x_{1}, y_{1}, z_{1}\right), S\left(x_{2}, y_{2}, z_{2}\right)\right), \\
& d\left(g y_{2}, g y_{3}\right) \leq k H\left(T\left(y_{1}, z_{1}, x_{1}\right), S\left(y_{2}, z_{2}, x_{2}\right)\right), \\
& d\left(g z_{2}, g z_{3}\right) \leq k H\left(T\left(z_{1}, x_{1}, y_{1}\right), S\left(z_{2}, x_{2}, y_{2}\right)\right) .
\end{aligned}
$$

Continuing this process, we obtain sequences $\left\{x_{n}\right\},\left\{y_{n}\right\}$, and $\left\{z_{n}\right\}$ in $X$ as $g x_{2 n+1} \in S\left(x_{2 n}, y_{2 n}, z_{2 n}\right), g y_{2 n+1} \in$ $S\left(y_{2 n}, z_{2 n}, x_{2 n}\right), g z_{2 n+1} \in S\left(z_{2 n}, x_{2 n}, y_{2 n}\right)$, and $g x_{2 n+2} \in$ $T\left(x_{2 n+1}, y_{2 n+1}, z_{2 n+1}\right), g y_{2 n+2} \in T\left(y_{2 n+1}, z_{2 n+1}, x_{2 n+1}\right)$, $g z_{2 n+2} \in T\left(z_{2 n+1}, x_{2 n+1}, y_{2 n+1}\right)$ such that

$$
\begin{aligned}
& d\left(g x_{2 n+1}, g x_{2 n+2}\right) \\
& \quad \leq k H\left(S\left(x_{2 n}, y_{2 n}, z_{2 n}\right), T\left(x_{2 n+1}, y_{2 n+1}, z_{2 n+1}\right)\right), \\
& d\left(g y_{2 n+1}, g y_{2 n+2}\right) \\
& \quad \leq k H\left(S\left(y_{2 n}, z_{2 n}, x_{2 n}\right), T\left(y_{2 n+1}, z_{2 n+1}, x_{2 n+1}\right)\right), \\
& d\left(g z_{2 n+1}, g z_{2 n+2}\right) \\
& \quad \leq k H\left(S\left(z_{2 n}, x_{2 n}, y_{2 n}\right), T\left(z_{2 n+1}, x_{2 n+1}, y_{2 n+1}\right)\right), \\
& d\left(g x_{2 n+2}, g x_{2 n+3}\right) \\
& \quad \leq k H\left(T\left(x_{2 n+1}, y_{2 n+1}, z_{2 n+1}\right), S\left(x_{2 n+2}, y_{2 n+2}, z_{2 n+2}\right)\right), \\
& d\left(g y_{2 n+2}, g y_{2 n+3}\right) \\
& \quad \leq k H\left(T\left(y_{2 n+1}, z_{2 n+1}, x_{2 n+1}\right), S\left(y_{2 n+2}, z_{2 n+2}, x_{2 n+2}\right)\right), \\
& d\left(g z_{2 n+2}, g z_{2 n+3}\right) \\
& \quad \leq k H\left(T\left(z_{2 n+1}, x_{2 n+1}, y_{2 n+1}\right), S\left(z_{2 n+2}, x_{2 n+2}, y_{2 n+2}\right)\right) .
\end{aligned}
$$


From (58), we have

$$
\begin{aligned}
& d\left(g x_{2 n+1}, g x_{2 n+2}\right) \\
& \leq k H\left(S\left(x_{2 n}, y_{2 n}, z_{2 n}\right), T\left(x_{2 n+1}, y_{2 n+1}, z_{2 n+1}\right)\right) \\
& \leq \sqrt{h} \max \left\{d\left(g x_{2 n}, g x_{2 n+1}\right), d\left(g y_{2 n}, g y_{2 n+1}\right)\right. \text {, } \\
& d\left(g z_{2 n}, g z_{2 n+1}\right), d\left(S\left(x_{2 n}, y_{2 n}, z_{2 n}\right), g x_{2 n}\right), \\
& d\left(S\left(x_{2 n}, y_{2 n}, z_{2 n}\right), g x_{2 n}\right), \\
& \left(T\left(x_{2 n+1}, y_{2 n+1}, z_{2 n+1}\right), g x_{2 n+1}\right. \\
& \left.\left.+d\left(S\left(x_{2 n}, y_{2 n}, z_{2 n}\right), g x_{2 n}\right)\right) \frac{1}{2}\right\} \\
& \leq \sqrt{h} \max \left\{d\left(g x_{2 n}, g x_{2 n+1}\right), d\left(g y_{2 n}, g y_{2 n+1}\right)\right. \text {, } \\
& d\left(g z_{2 n}, g z_{2 n+1}\right), d\left(g x_{2 n+1}, g x_{2 n}\right), \\
& d\left(g x_{2 n+1}, g x_{2 n}\right),\left(d\left(g x_{2 n+2}, g x_{2 n+1}\right)\right. \\
& \left.\left.+d\left(g x_{2 n+1}, g x_{2 n}\right)\right) \frac{1}{2}\right\} \\
& \leq \sqrt{h} \max \left\{d\left(g x_{2 n-1}, g x_{2 n}\right), d\left(g y_{2 n-1}, g y_{2 n}\right)\right. \text {, } \\
& d\left(g x_{2 n}, g x_{2 n+1}\right) \\
& \left.d\left(g z_{2 n-1}, g z_{2 n}\right)\right\}, \\
& \leq \sqrt{h} \max \left\{d\left(g x_{2 n-1}, g x_{2 n}\right), d\left(g y_{2 n-1}, g y_{2 n}\right),\right. \\
& d\left(g z_{2 n-1}, g z_{2 n}\right), d\left(g x_{2 n}, g x_{2 n-1}\right), \\
& d\left(g x_{2 n+1}, g x_{2 n}\right) \text {, } \\
& \left.\frac{d\left(g x_{2 n}, g x_{2 n-1}\right)+d\left(g x_{2 n+1}, g x_{2 n}\right)}{2}\right\} \\
& \left.d\left(g z_{2 n-1}, g z_{2 n}\right), d\left(g x_{2 n+1}, g x_{2 n}\right)\right\} \text {. }
\end{aligned}
$$

Hence, if we suppose that $d\left(g x_{2 n}, g x_{2 n+1}\right) \leq$ $\sqrt{h} d\left(g x_{2 n+1}, g x_{2 n}\right)$, then

$$
d\left(g x_{2 n}, g x_{2 n+1}\right)=0 \text {. }
$$

Therefore,

$$
\begin{gathered}
d\left(g x_{2 n}, g x_{2 n+1}\right) \\
\leq \sqrt{h} \max \left\{d\left(g x_{2 n-1}, g x_{2 n}\right), d\left(g y_{2 n-1}, g y_{2 n}\right),\right. \\
\left.d\left(g z_{2 n-1}, g z_{2 n}\right)\right\} .
\end{gathered}
$$

Similarly, we obtain

$$
\begin{aligned}
& d\left(g y_{2 n}, g y_{2 n+1}\right) \\
& \leq \sqrt{h} \max \left\{d\left(g y_{2 n-1}, g y_{2 n}\right), d\left(g z_{2 n-1}, g z_{2 n}\right),\right. \\
& \left.d\left(g x_{2 n-1}, g x_{2 n}\right)\right\}, \\
& d\left(g z_{2 n}, g z_{2 n+1}\right) \\
& \leq \sqrt{h} \max \left\{d\left(g z_{2 n-1}, g z_{2 n}\right), d\left(g x_{2 n-1}, g x_{2 n}\right),\right. \\
& \left.d\left(g y_{2 n-1}, g y_{2 n}\right)\right\} .
\end{aligned}
$$

Using (65) and (66), we obtain for all $n \in \mathbb{N}$

$$
\begin{aligned}
& d\left(g x_{n}, g x_{n+1}\right) \leq(\sqrt{h})^{n} \delta, \\
& d\left(g y_{n}, g y_{n+1}\right) \leq(\sqrt{h})^{n} \delta, \\
& d\left(g z_{n}, g z_{n+1}\right) \leq(\sqrt{h})^{n} \delta,
\end{aligned}
$$

where $\delta=\max \left\{d\left(g x_{0}, g x_{1}\right), d\left(g y_{0}, g y_{1}\right), d\left(g z_{0}, g z_{1}\right)\right\}$.Thus for $m, n \in N$ with $m>n$.

$$
\begin{aligned}
& d\left(g x_{n}, g x_{m+n}\right) \\
& \leq d\left(g x_{n}, g x_{n+1}\right)+d\left(g x_{n+1}, g x_{n+2}\right) \\
&+\cdots+d\left(g x_{n+m-1}, g x_{n+m}\right) \\
& \leq(\sqrt{h})^{n} \delta+(\sqrt{h})^{n+1} \delta+\cdots+(\sqrt{h})^{n+m-1} \delta \\
& \leq \sum_{i=n}^{m+n-1}(\sqrt{h})^{i} \delta .
\end{aligned}
$$

Hence we conclude that $\left\{g x_{n}\right\}$ is a Cauchy sequence in $g(X)$. Similarly, we obtain that $\left\{g y_{n}\right\}$ and $\left\{g z_{n}\right\}$ are Cauchy sequences in $g(X)$. Since $g(X)$ is complete, there exists $x, y, z \in X$ such that $g x_{n} \rightarrow x, g y_{n} \rightarrow y$, and $g z_{n} \rightarrow z$. Thus from (58),

$d(T(x, y, z), g x)$

$$
\begin{aligned}
& \leq d\left(T(x, y, z), g x_{2 n+1}\right)+d\left(g x_{2 n+1}, g x\right) \\
& \leq H\left(T(x, y, z), S\left(x_{2 n}, y_{2 n}, z_{2 n}\right)\right)+d\left(g x_{2 n+1}, g x\right) \\
& \leq h \max \left\{d\left(g x, g x_{2 n}\right), d\left(g y, g y_{2 n}\right), d\left(g z, g z_{2 n}\right),\right. \\
& d(T(x, y, z), g x), d\left(S\left(x_{2 n}, y_{2 n}, g z_{2 n}\right), g x_{2 n}\right), \\
& \left(d\left(T(x, y, z), g x_{2 n}\right)\right. \\
& \left.\left.+d\left(S\left(x_{2 n}, y_{2 n}, g z_{2 n}\right), g x\right)\right) \frac{1}{2}\right\} \\
& +d\left(g x_{2 n+1}, g x\right)
\end{aligned}
$$




$$
\begin{gathered}
\leq h \max \left\{d\left(g x, g x_{2 n}\right), d\left(g y, g y_{2 n}\right), d\left(g z, g z_{2 n}\right),\right. \\
d(T(x, y, z), g x), d\left(g x_{2 n+1}, g x_{2 n}\right), \\
\left.\frac{d\left(T(x, y, z), g x_{2 n}\right)+d\left(g x_{2 n+1}, g x\right)}{2}\right\} \\
+d\left(g x_{2 n+1}, g x\right) .
\end{gathered}
$$

On taking limit as $n \rightarrow \infty$, we get

$$
d(T(x, y, z), g x) \leq h d(T(x, y, z), g x),
$$

which implies that

$$
d(T(x, y, z), g x)=0 .
$$

As $T(x, y, z)$ is closed, $g x \in T(x, y, z)$. Similarly, $g y \in$ $T(y, z, x), g z \in T(z, x, y)$. Therefore $(x, y, z)$ is a tripled coincidence point of $T$ and $g$. Similarly, $(x, y, z)$ is a tripled coincidence point of $S$ and $g$. Thus $(x, y, z)$ is a tripled coincidence point of $(S, g)$ and $(T, g)$. Suppose that (i) holds; then, for some $(x, y, z) \in \Upsilon(g, S)$ and $\Upsilon(g, T)$, we have $g x \in$ $S(x, y, z), g y \in S(y, z, x), g z \in S(z, x, y)$ and $g x \in T(x, y, z)$, $g y \in T(y, z, x), g z \in S(z, x, y)$. Since $(S, g)$ and $(T, g)$ are $\mathrm{w}$-compatible, we have

$$
\begin{aligned}
& g(S(x, y, z)) \subseteq S(g x, g y, g z), \\
& g(T(x, y, z)) \subseteq T(g x, g y, g z),
\end{aligned}
$$

for $(x, y, z) \in \Upsilon(g, S)$ and $\Upsilon(g, T)$. Since $g x \in S(x, y, z), g y \in$ $S(y, z, x)$, and $g z \in S(z, x, y)$. So $g^{2} x \in S(g x, g y, g z), g^{2} y \in$ $S(g y, g z, g x)$, and $g^{2} z \in S(g z, g x, g y)(\Rightarrow(g x, g y, g z) \in$ $\Upsilon(g, S))$. Similarly $g^{2} x \in T(g x, g y, g z), g^{2} y \in T(g y, g z, g x)$ and $g^{2} z \in T(g z, g x, g y),(\Rightarrow(g x, g y, g z) \in \Upsilon(g, T))$. Continuing in this way, we get

$$
\begin{aligned}
& \left(g^{n-1} x, g^{n-1} y, g^{n-1} z\right) \in \Upsilon(g, S), \\
& \left(g^{n-1} x, g^{n-1} y, g^{n-1} z\right) \in \Upsilon(g, T)
\end{aligned}
$$

for all $n \geq 1$ and

$$
\begin{aligned}
& g^{n} x \in S\left(g^{n-1} x, g^{n-1} y, g^{n-1} z\right), \\
& g^{n} x \in T\left(g^{n-1} x, g^{n-1} y, g^{n-1} z\right) .
\end{aligned}
$$

And $\lim _{n \rightarrow \infty} g^{n} x=u, \lim _{n \rightarrow \infty} g^{n} y=v$ and $\lim _{n \rightarrow \infty} g^{n} z=$ $w$ for $(x, y, z) \in \Upsilon(S, g)$ and $(x, y, z) \in \Upsilon(T, g)$. Since $g$ is continuous at $u, v$, and $w$, we have $u=g u, v=g v$, and $w=$ $g w$. Now using (58), we get

$$
\begin{aligned}
& d(g u, S(u, v, w)) \\
& \leq d\left(g u, g^{n} x\right)+d\left(g^{n} x, S(u, v, w)\right) \\
& \leq d\left(g u, g^{n} x\right) \\
& +H\left(T\left(g^{n-1} x, g^{n-1} y, g^{n-1} z\right), S(u, v, w)\right) \\
& \leq d\left(g u, g^{n} x\right) \\
& +h \max \left\{d\left(g^{n} x, g u\right), d\left(g^{n} y, g v\right), d\left(g^{n} z, g w\right),\right. \\
& d\left(T\left(g^{n-1} x, g^{n-1} y, g^{n-1} z\right), g^{n} x\right), \\
& d(S(u, v, w), g u), \\
& \left(d\left(T\left(g^{n-1} x, g^{n-1} y, g^{n-1} z\right), g^{n} x\right)\right. \\
& \left.+d(S(u, v, w), g u)) \frac{1}{2}\right\}, \\
& \leq d\left(g u, g^{n} x\right) \\
& +h \max \left\{d\left(g^{n} x, g u\right), d\left(g^{n} y, g v\right), d\left(g^{n} z, g w\right),\right. \\
& d\left(g^{n} x, g^{n} x\right), d(S(u, v, w), g u), \\
& \left.\frac{d\left(g^{n} x, g^{n} x\right)+d(S(u, v, w), g u)}{2}\right\} .
\end{aligned}
$$

Taking limit as $n \rightarrow \infty$, we get

$$
d(g u, S(u, v, w)) \leq h d(S(u, v, w), g u) .
$$

Hence

$$
d(g u, S(u, v, w))=0
$$

and therefore

$$
g u \in S(u, v, w) .
$$

Similarly,

$$
g v \in S(v, w, u), \quad g w \in S(w, u, v) .
$$

Consequently,

$$
\begin{gathered}
u=g u \in S(u, v, w), \quad v=g v \in S(v, w, u), \\
w=g w \in S(w, u, v) .
\end{gathered}
$$

Similarly,

$$
\begin{gathered}
u=g u \in T(u, v, w), \quad v=g v \in T(v, w, u), \\
w=g w \in T(w, u, v) .
\end{gathered}
$$


Hence $(u, v, w)$ is a tripled common fixed point of $(S, g)$ and $(T, g)$. Now suppose that (ii) holds. Since $g$ is $S, T$ idempotent for some $(x, y, z) \in \Upsilon(g, S)$ and $(x, y, z) \in$ $\Upsilon(g, T)$, we have $g^{2} x \in S(g x, g y, g z), g^{2} y \in S(g y, g z, g x)$, $g^{2} z \in S(g z, g x, g y)$, and $g^{2} x \in T(g x, g y, g z), g^{2} y \in$ $T(g y, g z, g x), g^{2} z \in T(g z, g x, g y)$. Since we have $g^{2} x=$ $g x, g^{2} y=g y$ and $g^{2} z=g z$. So,

$$
\begin{aligned}
& g x=g^{2} x \in S(g x, g y, g z), \\
& g y=g^{2} y \in S(g y, g z, g x), \\
& g z=g^{2} z \in S(g z, g x, g y), \\
& g x=g^{2} x \in T(g x, g y, g z), \\
& g y=g^{2} y \in T(g y, g z, g x), \\
& g z=g^{2} z \in T(g z, g x, g y) .
\end{aligned}
$$

Hence $(g x, g y, g z)$ is a tripled common fixed point of $(S, g)$ and $(T, g)$. Now suppose that (iii) holds. For some $(x, y, z) \in$ $\Upsilon(g, S)$ and $(x, y, z) \in \Upsilon(g, T)$, we get

$$
\begin{array}{ll}
g x \in S(x, y, z), & g y \in S(y, w, x), \\
g z \in S(z, x, y), & g x \in T(x, y, z), \\
g y \in T(y, w, x), & g z \in T(z, x, y) .
\end{array}
$$

Since $g$ is continuous at $x$ and $y$, we get $g x=x, g y=y$, and $g z=z$. Thus

$$
\begin{array}{ll}
x=g x \in S(x, y, z), & y=g y \in S(y, w, x), \\
z=g z \in S(z, x, y), & x=g x \in T(x, y, z), \\
y=g y \in T(y, w, x), & z=g z \in T(z, x, y) .
\end{array}
$$

This implies that $(x, y, z)$ is a tripled common fixed point of $(S, g)$ and $(T, g)$.

Conclusion. In this paper, we established tripled coincidence and common fixed point results for a pair of hybrid mappings in the context of complete metric spaces. These results are extensions of results in $[4,5,11]$ to the case tripled coincidence and common fixed points. Our results may be the motivation to other authors for extending and improving these results to be suitable tools for their applications.

\section{Conflict of Interests}

The authors declare that they have no competing interests.

\section{Authors' Contribution}

All authors contributed equally and significantly to writing this paper. All authors read and approved the final paper.

\section{Acknowledgment}

Marwan Amin Kutbi gratefully acknowledges the support from the Deanship of Scientific Research (DSR) at King Abdulaziz University (KAU) during this research.

\section{References}

[1] S. B. Nadler Jr., "Multi-valued contraction mappings," Pacific Journal of Mathematics, vol. 30, pp. 475-488, 1969.

[2] B. C. Dhage, "Hybrid fixed point theory for strictly monotone increasing multi-valued mappings with applications," Computers \& Mathematics with Applications, vol. 53, no. 5, pp. 803-824, 2007.

[3] B. C. Dhage, "A general multi-valued hybrid fixed point theorem and perturbed differential inclusions," Nonlinear Analysis: Theory, Methods \& Applications, vol. 64, no. 12, pp. 2747-2772, 2006.

[4] T. Gnana Bhaskar and V. Lakshmikantham, "Fixed point theorems in partially ordered metric spaces and applications," Nonlinear Analysis: Theory, Methods \& Applications, vol. 65, no. 7, pp. 1379-1393, 2006.

[5] V. Lakshmikantham and L. Cirić, "Coupled fixed point theorems for nonlinear contractions in partially ordered metric spaces," Nonlinear Analysis: Theory, Methods \& Applications, vol. 70, no. 12, pp. 4341-4349, 2009.

[6] E. Karapınar, "Couple fixed point theorems for nonlinear contractions in cone metric spaces," Computers \& Mathematics with Applications, vol. 59, no. 12, pp. 3656-3668, 2010.

[7] V. Berinde and M. Borcut, "Tripled fixed point theorems for contractive type mappings in partially ordered metric spaces," Nonlinear Analysis: Theory, Methods \& Applications, vol. 74, no. 15, pp. 4889-4897, 2011.

[8] M. Borcut and V. Berinde, "Tripled coincidence theorems for contractive type mappings in partially ordered metric spaces," Applied Mathematics and Computation, vol. 218, no. 10, pp. 5929-5936, 2012.

[9] N. Hussain, A. Latif, and M. H. Shah, "Coupled and tripled coincidence point results without compatibility," Fixed Point Theory and Applications, vol. 2013, article 77, 9 pages, 2013.

[10] N. Hussain and A. Alotaibi, "Coupled coincidences for multivalued contractions in partially ordered metric spaces," Fixed Point Theory and Applications, vol. 2011, article 82, 15 pages, 2011.

[11] D. Ilić, M. Abbas, and M. A. Khan, "Coupled coincidence point and coupled common fixed point theorems in partially ordered metric spaces with $\omega$-distance," Fixed Point Theory and Applications, vol. 2010, Article ID 134897, 11 pages, 2010.

[12] M. Abbas, A. R. Khan, and T. Nazir, "Coupled common fixed point results in two generalized metric spaces," Applied Mathematics and Computation, vol. 217, no. 13, pp. 6328-6336, 2011.

[13] H. Aydi, M. Abbas, and M. Postolache, "Coupled coincidence points for hybrid pair of mappings via mixed monotone property," Journal of Advanced Mathematical Studies, vol. 5, no. 1, pp. 118-126, 2012.

[14] I. Beg and A. Azam, "Fixed points of asymptotically regular multivalued mappings," Journal of the Australian Mathematical Society. Series A, vol. 53, no. 3, pp. 313-326, 1992. 
[15] Y. J. Cho, M. H. Shah, and N. Hussain, "Coupled fixed points of weakly $F$-contractive mappings in topological spaces," Applied Mathematics Letters, vol. 24, no. 7, pp. 1185-1190, 2011.

[16] M. A. Kutbi, A. Azam, J. Ahmad, and C. Di Bari, "Some common coupled fixed point results for generalized contraction in complex-valued metric spaces," Journal of Applied Mathematics, vol. 2013, Article ID 352927, 10 pages, 2013. 


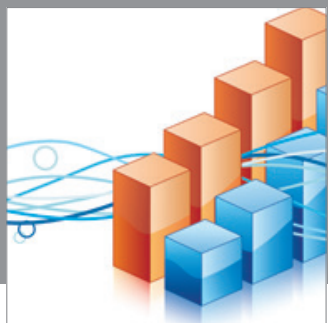

Advances in

Operations Research

mansans

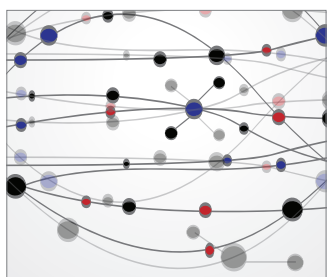

The Scientific World Journal
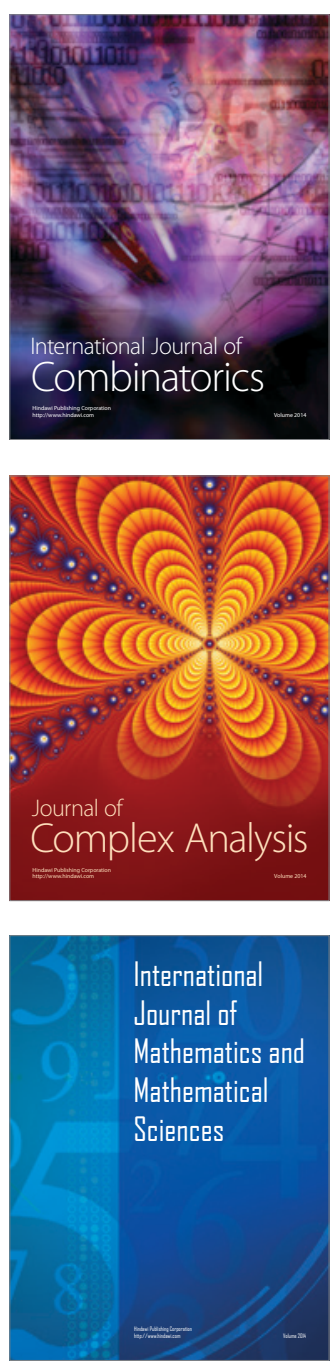
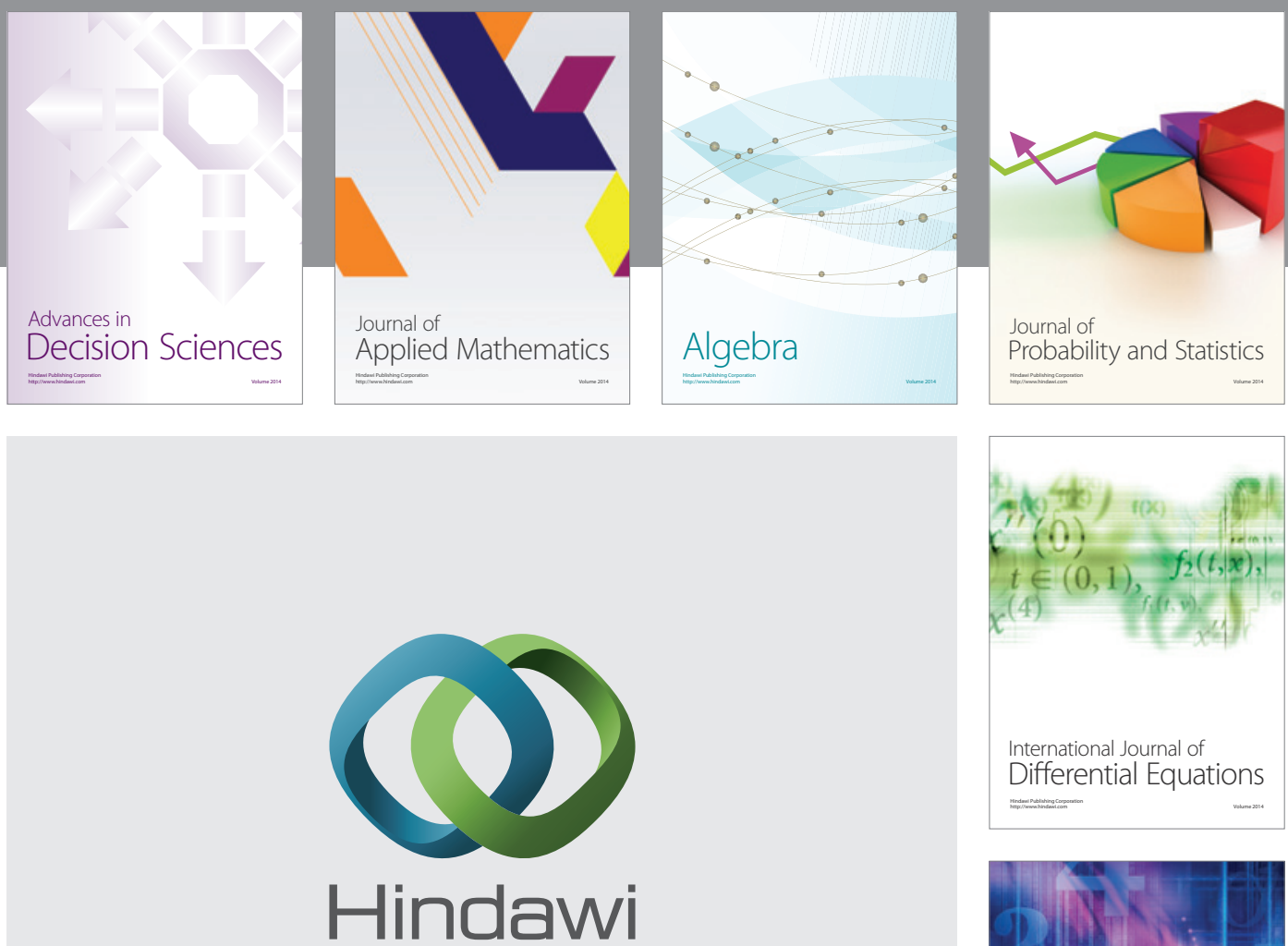

Submit your manuscripts at http://www.hindawi.com
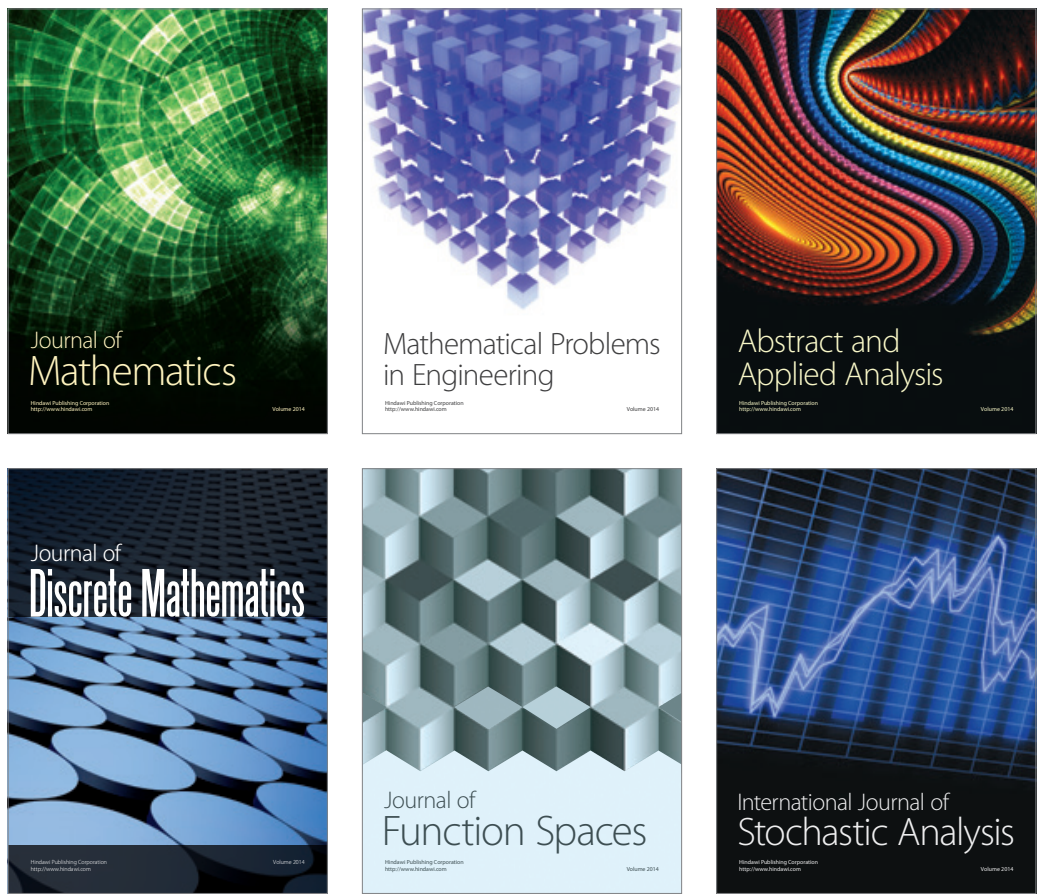

Journal of

Function Spaces

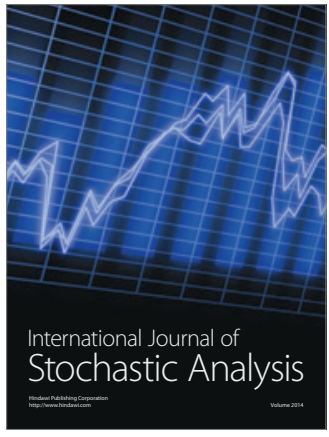

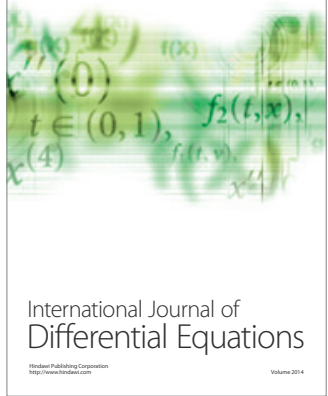
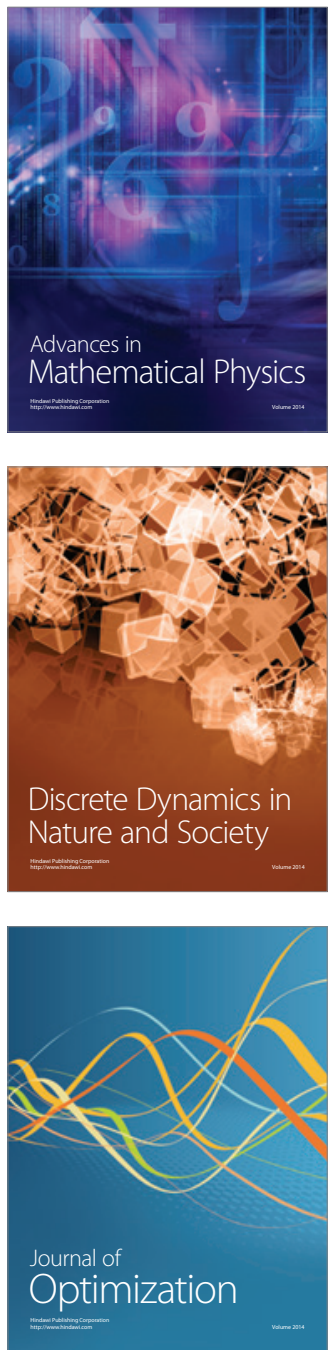